\title{
Image-Guided Pre-Operative Localisation of Impalpable Breast Lesions: Analysis of the Hook-Wire Localisation Technique
}

\author{
Alya Binmahfouz ${ }^{1,2}$, Marita K Prior*1, ${ }^{1}$ Sai Navya Purchuri ${ }^{1}$ and Shohreh Rezai ${ }^{1}$ \\ ${ }^{1}$ Department of Medical Imaging, Royal Brisbane and Women's Hospital, Australia
}

${ }^{2}$ Department of Medical Imaging, King Abdul-Aziz University Hospital, Saudi Arabia

Received: March 15, 2018; Published: March 28, 2018

*Corresponding author: Marita K Prior, Department of Medical Imaging, Royal Brisbane and Women's Hospital, Brisbane, QLD, Australia, Tel: 073646 7225; Email: Marita.Prior@health.qld.gov.au

Abstract

Introduction: Hook-wire localisation (HWL) under sonographic, tomosynthesis or MRI guidance is the most common technique used to localize impalpable breast lesions. We retrospectively review and discuss HWL success and complication rate, and the number of breast lesions that required re-excision at our institution. We compare our results with other centres, identifying alternative methods for localizing impalpable breast lesions.

Methods: The medical records of 141 patients who underwent HWL at our institution over a 36 month period were retrospectively reviewed. Only impalpable lesions were targeted. Post-surgical specimen radiography was performed to verify retrieval of the target and wire, and to radio logically assess surgical margins. Surgical margins were deemed positive if malignancy extended to the edge of specimen, and close if malignancy extended to less than $3 \mathrm{~mm}$.

Results: Altogether 40 patients had tomosynthesis-guided HWL and 101 patients had ultrasound-guided HWL. 106 excisions were therapeutic and 35 were diagnostic. Pathology identified 115 lesions as malignant, 6 as high-risk and 20 as benign lesions. Seven patients had complications related to the hook-wire. Post-localisation waiting time ranged from 1-10 hours (median, 4.5 hours). 52 patients required re-excision due to involved margins and of these patients, 18 had a complete mastectomy. Conclusion: Hook-wire localisation is a valuable technique for targeting breast lesions; however, direct and indirect complications associated with this technique do exist. In accordance with the findings of this study, and the literature, it would be of value for our clinicians to be aware of these complications and to consider alternative, more reliable localisation techniques.

Keywords: Breast carcinoma; Lobular; Ductal; Mammography; Tomo synthesis; Hook-wire localization

Abbreviations: HWL: Hook-Wire Localisation; RBWH: Royal Brisbane \& Women’s Hospital; DCIS: Ductal Carcinoma In Situ; EIC: Extensive Intra-ductal Component; RSL: Radioactive Seed Localisation; ROLL: Radio-Guided Occult Lesion Localisaion; IOUS: Labelled Colloid and IntraOperative Ultra-Sound

\section{Introduction}

Breast cancer is one of the leading causes of death in women1. In 2007 it was second most common cause of cancer death among Australian women. In recent years, owing to widespread breast screening programs, early detection of breast cancer is currently more feasible than before. As a result, impalpable breast lesions nowadays constitute a significant proportion of breast lesions (up to $25 \%$ ) 2, 3, which subsequently require image guided localisation to facilitate surgical excision. Up to $20 \%$ of impalpable lesions are malignant4. The most widely used technique of localisation is hookwire localisation (HWL), which we routinely use in our institution, the Royal Brisbane \& Women's Hospital (RBWH). This paper aims to investigate the current procedure of HWL at our institution with a view to identifying common issues that arise with this technique. Ultimately, we want to improve the outcomes of our patients.

\section{Methods}

This retrospective study included all patients who underwent HWL at the RBWH, Brisbane, Queensland, Australia, from January 2013 to December 2015. The study was approved by the local ethics committee at the RBWH. We accessed the hospital database to collect the study population. Radiologic and pathologic results were recorded from the electronic medical record. A total of 141 
pre-operatives HWL were performed using Hawkins TM III and D Wire (Breast Localization Needles by Argon Medical Devices). All targeted lesions were impalpable. Palpable lesions were excluded. We also excluded cases that were diagnosed in our institution but where the surgical procedures were performed at another centre. Cases that required re-excision but were performed at another centre were also excluded from our study. All HWL were performed on the day of surgery by five radiologists under either tomosynthesis (Figure 1) or ultrasound (Figure 2) guidance, according to the type of lesion localised. Post-localisation 2-view mammogram was performed to document position of the wire (see Supporting Information Figure 1). Post-surgical specimen radiography was performed to verify retrieval of the target and wire, and to radio logically assess surgical margins. Surgical margins were deemed positive if malignancy extended to the edge of specimen, and close if malignancy extended to less than $3 \mathrm{~mm}$.

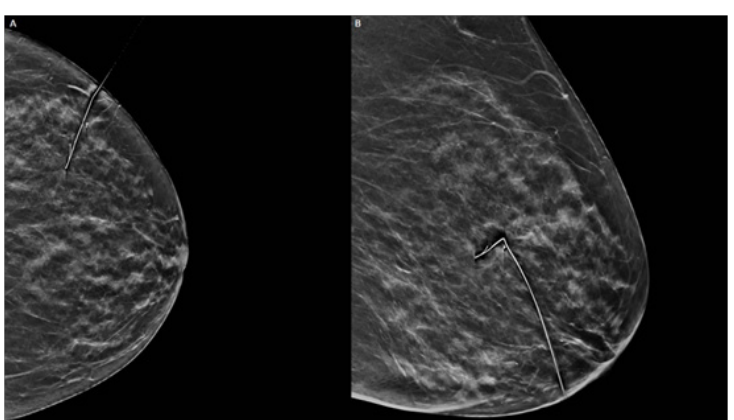

Figure 1: Tomo synthesis-guided hook-wire localisation of the left breast A) cranio-caudal and B) lateral views show localisation of a post biopsy marker clip at the upper outer quadrant.

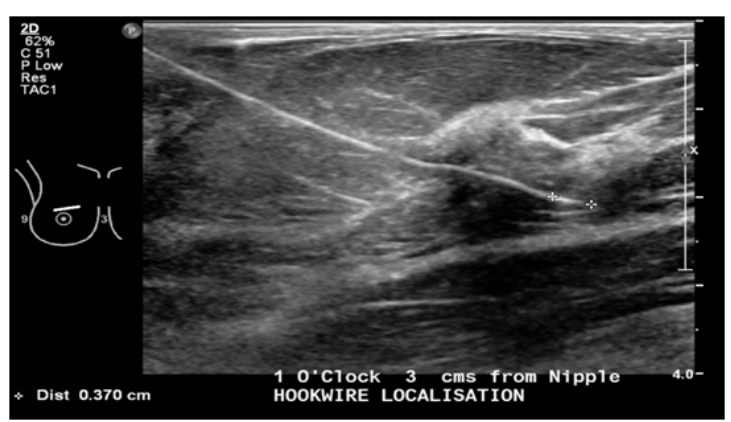

Figure 2: Transverse grey scale ultrasound image shows hook-wire localisation of a solid mass in the right breast at $1 \mathrm{o}^{\prime}$ clock, $3 \mathrm{~cm}$ from the nipple.

\section{Results}

Our study population included 141 females, between 31-82 years of age (mean age of 57 years). Of this cohort of female patients, 40 patients $(28.3 \%)$ had tomosynthesis guided HWL, while 101 patients $(71.6 \%)$ had ultrasound guided HWL. 106 excisions were therapeutic and 35 were diagnostic. 115/141 (81.5\%) lesions were malignant, 6/141 (4.3\%) were high-risk lesions, and 20/141 (14.1\%) were benign lesions. Final histopathology results showed ductal carcinoma in situ (DCIS) in 27 patients, invasive ductal carcinoma (IDC) in 28 patients, DCIS and IDC in 39 patients, lobular cancer in 13 patients, mixed ductal and lobular cancer in 5 patients, angiosarcoma in one patient, metaplastic carcinoma in 1 patient, and melanoma metastasis in 1 patient (Table 1) (Appendix). The targeted lesions varied among: 103 (73\%) non-calcified masses, $24(17 \%)$ clustered micro calcifications, $1(0.7 \%)$ mass with calcifications, $1(0.7 \%)$ architectural distortion, and 12 (8.5\%) post biopsy marker clips (Table 2). On our initial imaging reports, 1/141 $(0.7 \%)$ was reported as BI-RADS 6, 110/141 (78\%) BI-RADS 5, 25/141 (17.7\%) BI-RADS 4, and 5/141 (3.5\%) BI-RADS 3.

Table 1: Malignant final histopathology diagnosis versus imaging-guided HWL.

\begin{tabular}{|c|c|c|c|}
\hline $\begin{array}{c}\text { Final Pathology } \\
\text { Diagnosis }\end{array}$ & $\begin{array}{c}\text { Tomo } \\
\text { synthesis HWL }\end{array}$ & $\begin{array}{c}\text { Ultrasound } \\
\text { HWL }\end{array}$ & Total (\%) \\
\hline DCIS & 21 & 6 & $27(19 \%)$ \\
\hline IDC & 1 & 27 & $28(19.8 \%)$ \\
\hline DCIS and IDC & 9 & 30 & $39(27.6 \%)$ \\
\hline Lobular cancer & 4 & 9 & $13(9.2 \%)$ \\
\hline $\begin{array}{c}\text { Mixed ductal/ } \\
\text { lobular cancer }\end{array}$ & 2 & 3 & $5(3.5 \%)$ \\
\hline Angiosarcoma & 0 & 1 & $1(0.7 \%)$ \\
\hline $\begin{array}{c}\text { Metaplastic } \\
\text { carcinoma }\end{array}$ & 0 & 1 & $1(0.7 \%)$ \\
\hline $\begin{array}{c}\text { Metastatic } \\
\text { Melanoma }\end{array}$ & 0 & 1 & $1(0.7 \%)$ \\
\hline
\end{tabular}

Table 2: Types of lesions identified upon diagnosing an impalpable mass.

\begin{tabular}{|c|c|c|c|}
\hline & \multicolumn{3}{|c|}{ Modality } \\
\hline Lesion type & $\begin{array}{c}\text { Tomosynthesis- } \\
\text { guided }\end{array}$ & $\begin{array}{c}\text { Ultrasound- } \\
\text { guided }\end{array}$ & $\begin{array}{c}\text { Total } \\
\text { (percentage) }\end{array}$ \\
\hline $\begin{array}{c}\text { Non-calcified } \\
\text { mass }\end{array}$ & 3 & 100 & $103(73 \%)$ \\
\hline $\begin{array}{c}\text { Clustered micro } \\
\text { calcifications }\end{array}$ & 24 & 0 & $24(17 \%)$ \\
\hline Post-biopsy clip & 12 & 0 & $12(8.5 \%)$ \\
\hline $\begin{array}{c}\text { Mass with } \\
\text { calcifications }\end{array}$ & 1 & 0 & $1(0.7 \%)$ \\
\hline $\begin{array}{c}\text { Architectural } \\
\text { distortion }\end{array}$ & 0 & 1 & $1(0.7 \%)$ \\
\hline
\end{tabular}

Targeting was successful in $98.5 \%$, as $2 / 141$ lesions were missed during HWL. 7/141 (4.9\%) patients had complications related to the wire, $4(57.1 \%)$ of which were tomosynthesis guided, and 3 (42.9\%) were ultrasound guided. Post localisation waiting time ranged from 1-10 hours with a median of 4.5 hours. No major surgical complications were reported. 52/141 (36.9\%) patients had re-excision due to involved margins, 35/52 (67.3\%) were ultrasound guided and 17/52 (32.7\%) were tomosynthesis guided (see Supporting Information Table 1). 51/52 (98\%) had further malignancy in re-excision, and only 1 patient had fibrocystic changes in the final histopathologic examination, with solid mass on ultrasound which was thought discordant with imaging findings, as such she elected to repeat the excision to exclude a missed tumor; the final re-excision histopathology showed no malignancy. Out of 52 patients, 18 underwent mastectomy for involved margins. 
Of the 7 patients who had wire-related complications, 6 had malignant final diagnosis, and 1 had a benign diagnosis. 3 underwent localisation of solid masses, 2 micro calcifications, and 2 marker clips. The maximum waiting time for these patients was 9 hours, and minimum waiting time was 4 hours. 4 patients experienced displacement/ dislodgement of the wire during postlocalisation mammography necessitating insertion of a second wire, 1 patient encountered deviation of the wire by scar tissue, 1 patient had an attempt of clip localisation under ultrasound guidance which missed the clip, that was subsequently correctly localised under tomosynthesis guidance, and 1 patient had Intraoperative dislodgement of the wire (Table 3). In the present study, the radiologic-pathologic discordance rate was 3.5\%. 3 lesions were categorized as BI-RADS 4 due to suspicious calcifications on mammography, with final histopathologic diagnosis revealed fibrocystic changes (FCC); and 2 lesions were categorized as BIRADS 5 due to highly suspicious solid mass on sonography with final histopathologic diagnosis of FCC.

Table 3: Hook-wire related complications and the associated waiting time.

\begin{tabular}{|c|c|}
\hline \multicolumn{1}{|c|}{ Hook-wire related complications } & Waiting time \\
\hline 1. HW inadequately placed & 5 hours \\
\hline 2. HWL deviated due to scar tissue & 4 hours \\
\hline 3. HW dislodged & 6 hours \\
\hline 4. HW misplaced next to clip & 9 hours \\
\hline 5. HW dislodged & 9 hours \\
\hline 6. HW retracted & 5.5 hours \\
\hline 7. HW dislodged during surgery & 4 hours \\
\hline
\end{tabular}

*Statistical analysis of this table indicated that there was a probability that HW-related complication was attributable to the length of the waiting time ( $p$-value 0.03 ).

\section{Discussion}

HWL is considered by far the most popular and routinely used technique for localizing impalpable breast lesions, as well as, suspicious impalpable axillary lymph nodes. Our results show that 141 patients had a pre-operative hook-wire inserted to localize breast lesions at the RBWH over a three-year period. While the ages of these patients ranged from 31 years to 82 years, $93 \%$ of the patient cohort were above the age of 40 which is consistent with the age of women deemed 'at higher risk' of developing breast neoplasms as stated by the Breast Cancer in Australia Report of 20121. Accurate localisation of these lesions is paramount to ensuring the best possible outcomes for these patients. Radiological grading of the lesions, using the BI-RADS scale, indicated that 136 patients had either suspicious or highly suspicious lesions signifying the importance of accurately localizing the lesion, expedient excision and achieving clear margins. Over a third of our patient cohort (36.9\%) required re-excision due to involved margins. This percentage appears to be in a similar range to other hospitals.

At the Royal Perth Hospital in 2009, researchers found that $30 \%$ of the patient cohort receiving HWL for impalpable breast lesions required re-excision due to involved margins5. The researchers were able to explain that at least $11 \%$ of that cohort required re-excision due to both the radiologist and the surgeon underestimating the size of the tumour. There was the suggestion, however, that the remaining patients requiring re-excision might have done so due to the HWL technique although this would be difficult to validate. Other reported non-technical factors that increase risk of involved surgical margins include youth, small breast, diagnostic surgical biopsy, impalpable tumor, ductal carcinoma in situ (DCIS) or extensive intra-ductal component (EIC), use of adjuvant chemotherapy, large tumor size, and lobular histology6, 8. Pre-operative MRI examination is performed at some centres to evaluate full extent of tumor.

In this study $4.9 \%$ of the patients had a significant complication that was directly attributable to the HWL technique. These complications predominantly involved dislodgment of the wire during pre-operative waiting time. This period of time has been cited as a significant drawback of the HWL technique7, 9; the longer the patient waits, the greater the chance that the hook-wire becomes dislodged. We identified that all of the patients who had a wire that became dislodged had a significantly long waiting period (Table 3). Statistical analysis showed that the waiting time most likely contributed to the HW-related complication (p-value 0.03). Well known drawbacks of HWL include difficulty of placement in dense breasts, displacement of the wire during mammographic or surgical manipulation, excessive excision of normal breast tissue, minimal risk of pneumothorax related to wire migration, and patient's inconvenience with wire protruding out of the breast4, 7,9. Although rare, a case of wire migration into the supraclavicular fossa terminating in the posterior muscles of the neck was reported in AJR in 198810.

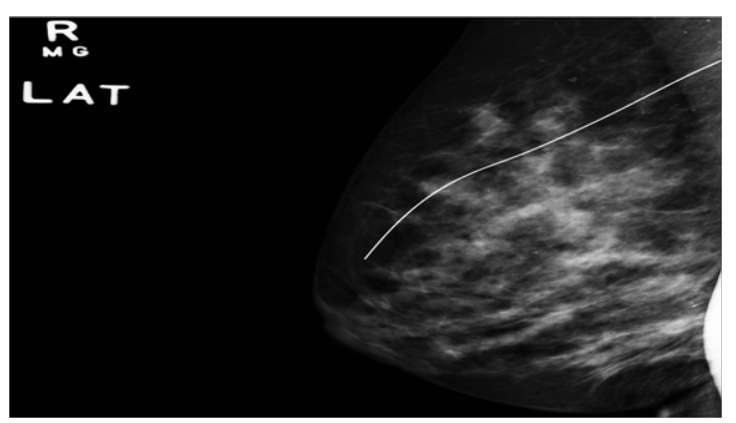

Figure 3: Post localisation lateral mammogram of the right breast show a hook-wire retracted into the breast and lodged into the pectoral muscle.

A similar incident occurred at our institution whereby a wire completely retracted into the pectoral muscle of a patient with a deeply situated lesion against the chest wall; no wire was visible outside the skin (Figures $3 \& 4$ ), complicating the surgical excision. Furthermore, the site of skin entry for the wire is not always consistent with the site of skin entry for the surgeon's incision, which may limit the surgeon's approach11. Finally, wire placement is done on the day of surgery, which may potentially cause conflicts between theatre and radiology schedules11. Consequently, alternative techniques have been developed, validated and 
proven to be superior to HWL. Techniques such as radioactive seed localisation (RSL) using 125I titanium seeds provide precise localisation, reduced procedure and operation time, improved rate of clear margins with reduced excision of healthy breast tissue, and higher acceptability by patients, surgeons, radiologists, and pathologists2-5, 11. Moreover, RSL performed more than one day in advance to surgery offers flexibility in scheduling localisation procedures and surgery, in addition to technical ease of post localisation mammograms without interference of wires. It can be performed at the same time as sentinel lymph node biopsy.

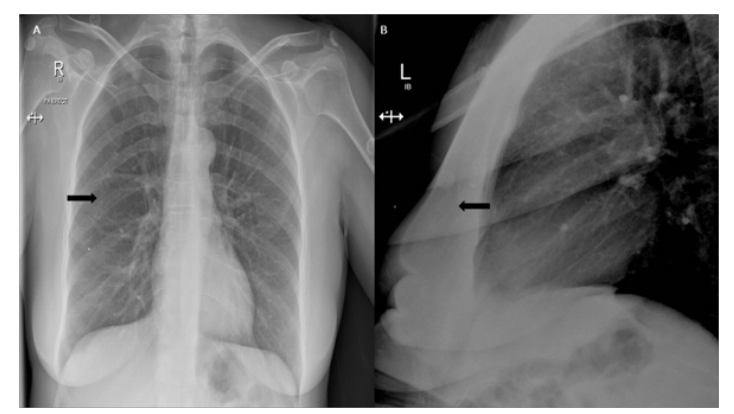

Figure 4: Frontal (A) and lateral (B) chest x-ray show a hook-wire (arrows) retracted into the right breast.

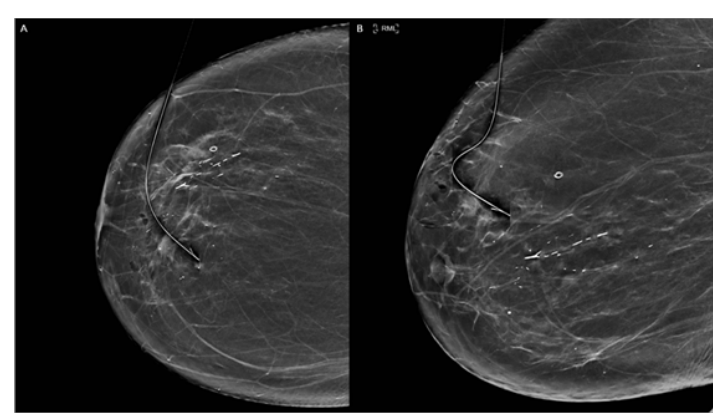

Figure 5.

Pathologists agree that sectioning of specimens and seed retrieval is technically easier than wires5. More importantly, the radiation dose to the patient, the public, and the medical team has been proved to be well below the permitted annual dose limit (1.5 versus $150 \mathrm{mSv}$ )3 and comparable to radiation dose in HWL11. This radio-guided localisation technique is widely used in the USA and Netherlands, and has recently been implemented in Western Australia12. A few adverse effects associated with RSL have been reported: intra-ductal dissemination of seed in intra-ductal lesions, and radiation down scatter from peri-areolar injection for SLN localisation hindering localisation of peri-areolar lesions. Occasionally the seed may stick to the tip of needle during deployment and may pull back with needle withdrawal, which is easily overcome by rotating the needle before withdrawal5.

Radio-guided occult lesion localisaion (ROLL) using 99mTclabelled colloid, and intra-operative ultrasound (IOUS) have been described as well, but neither have been readily accepted as common practice due to a number of associated shortcomings: short half-life of $99 \mathrm{mTc}$ precludes localisation in advance to surgery, difficulty of post-localisation confirmatory mammography because of non- opaque nature of colloid, and tracer diffusion away from injection site which decrease localisation accuracy5. Conversely, IOUS cannot be used for sonographically occult lesions, besides it requires trained surgeons and easy access to a portable US machine5.

\section{Conclusion}

While HWL is the most commonly used technique to localize impalpable breast lesions for surgery, this paper has identified significant complications as well as some limitations to surgical procedure. In our study the complications that could be directly attributed to the HWL technique was $4.9 \%$, however, a far higher percentage of our patients required re-excision which might indirectly be attributed to this technique. We also found that rate of complication increases when the wait time for surgery is longer. It is the aim of our institution to have no complications and to significantly reduce the number of patients that require reexcision. In accordance with previously published studies, radioguided occult lesion localisation using 125I seeds is a valuable and effective alternative to HWL which would reduce or eliminate some of the complications associated with wire localisation and improve surgical outcome.

\section{References}

1. (2012) Australian Institute of Health and Welfare \& Cancer Australia Breast Cancer in Australia: an overview. Cancer series 71(67): AIHW, Canberra, Australia.

2. Gray RJ, Pockaj BA, Karstaedt PJ, Roarke MC (2004) Radioactive seed localization of nonpalpable breast lesions is better than wire localization. Am J Surg 188(4): 377-380.

3. Taylor DB, Bourke AG, Westcott E, Burrage J, Latham B, et al. (2015) Radioguided occult lesion localisation using iodine- 125 seeds ('ROLLIS') for removal of impalpable breast lesions: First Australian experience. J Med Imaging Radiat Oncol 59(4): 411-420.

4. Chu TY, Lui CY, Hung WK, Kei SK, Choi CL, et al. (2010) Localisation of occult breast lesion: a comparative analysis of hook-wire and radioguided procedures. Hong Kong Med J 16(5): 367-372.

5. Davis PS, Wechsler RJ, Feig SA, March DE (1988) Migration of breast biopsy localization wire. AJR Am J Roentgenol 150(4): 787-788.

6. Ocal K, Dag A, Turkmenoglu O, Gunay EC, Yucel E, et al. (2011) Radioguided occult lesion localization versus wire-guided localization for non-palpable breast lesions: randomized controlled trial. Clinics 66(6): 1003-1007.

7. Dauer LT, Thornton C, Miodownik D, Boylan D, Holahan B, et al. (2013) Radioactive seed localization with ${ }^{125}$ I for non-palpable lesions prior to breast lumpectomy and/or excisional biopsy: methodology, safety, and experience of initial year. Health Phys 105(4): 356-365.

8. Jackson L, Bourke AG, AbdulAziz F, Taylor D (2014) Radioactive seed localisation to guide removal of impalpable lymph nodes (Radioguided Occult Lesion Localisation using Iodine-125 seeds, "ROLLIS"). BMJ Case Reports.

9. Jung W, Kang E, Kim SM, Kim D, Hwang Y, et al. (2012) Factors Associated with Re-excision after Breast-Conserving Surgery for Early-Stage Breast Cancer. J Breast Cancer 15(4): 412-419.

10. Jacobs L (2008) Positive Margins: The Challenge Continues for Breast Surgeons. Ann Surg Oncol 15(5): 1271-1272.

11. Rovera F, Frattini F, Marelli M, Corben AD, Vanoli C, et al. (2008) Radioguided occult lesion localization versus wire-guided localization in non-palpable breast lesions. International Journal of Surgery 6(6): S101-S103. 
12. Rampaul RS, Bagnall M, Burrell H, Pinder SE, Evans AJ, et al. (2004) Randomized clinical trial comparing radioisotope occult lesion localization and wire-guided excision for biopsy of occult breast lesions. Br J Surg 91(12): 1575-1577.

\section{(c) (i) This work is licensed under Creative}

Submission Link: https://biomedres.us/submit-manuscript.php

$\begin{array}{ll}\text { BIOMEDICAL } & \text { Assets of Publishing with us } \\ \text { RESEARCHES } & \text { - Global archiving of articles } \\ \text { - Immediate, unrestricted online access }\end{array}$

